\title{
EVOLUTION OF WHITE DWARFS AND THEIR PHYSICS
}

\author{
Giora Shaviv \\ Department of Physics and Astronomy \\ Tel Aviv University, Ramat Aviv, \\ Israel.
}

Introduction.

White Dwarfs (WD) are one of the fields in stellar structure in which the so-called "Input physics" is not yet completely clear today. In particular the equation of state (EOS) of WD affects directly one of the eminent problems in present day research of WD, namely is the "paucity" of low luminosity WD real? The reply depends in a sensitive way on the physical properties of the dense matter. For this reason we go in some detail into the physical problems of the "Input physics". We distinguish between the problem of the interfor-fully lonized matter and the envelope-partially lonized matter. Unfortunately space-time is not sufficient to cover in any detall the EOS of the envelope although this problem is extremely important to the evolution.

Transport properties depend on the structural form of the matter. We describe the improvements in the electron conductivity but do not discuss the fon buik viscosity which is also of interest to cooling, crystallizing WD's.

Finally, we discuss several processes that may affect the theoretical luminosity function (LF).

Energetics of cooling WD's. We follow closely Van Horn (1971) and Koester $(1978)$. Let $\left(p_{1}, T\right)$ denote the present state of a WD and $\left(p_{0}, 0\right)$ the final one. Evaluate all energies around the final state so that $E=E^{\circ}+\triangle E$ where $E^{O}=E\left(p_{0}, 0\right)$ and $\triangle E=E\left(p_{1}, T\right)-E^{O}$. The energies of the WD are: $E_{e f}$ - the perfect electron gas contribution, $E_{\text {- }}$ - the classIcal contribution of the ions. At high temperatures 1 tops $3 / 2 \mathrm{kT}$ per particle, at lower $T$ it 18 the phonon contribution and at $T=0$ it 18 the zero point energy of the lons. $E$ - the contribution of the Coulomb interactions (Debye-Huckel [DfGulat low $\Gamma$, bcc lattice at high $\Gamma$ ) together with all the corrections. Define now $a=E^{\circ} \operatorname{coul} / \mathrm{E}^{\circ}$ grav and $b=E^{\circ}$ ion $/ E^{\circ}$ grav. It can be shown that:

$$
\Delta E_{\text {binding }}=\Delta E_{\text {ion }}+\Delta E_{\text {coul }}-(a+3 \beta b) \Delta E_{\text {grav }}+\frac{\pi^{2}}{2} Z\left\langle\frac{k T}{\epsilon_{f}}\right\rangle N_{\text {ion }} k T(1)
$$

where $\beta=<P$ ion $/ U_{\text {ion }}>$, $U_{\text {ion }}$ is the energy density of the lons. The last term is the thermal energy of the electrons and is sometimes called the "gravitational energy release". $\Delta E_{g r a v}$ depends only on $\delta \rho$. 
The first term can be written as: $\Delta E_{10 n}=\Delta E_{\text {ion, } \rho}+\Delta E_{\text {ion, } T}$ where the first term is the change due to density only, while the second is due to temperature change only. The change in the binding energy then becomes:

$$
\Delta E_{\text {binding }}=\Delta E_{\text {ion, } T}+\Delta E_{\text {coul }, T}+\frac{\pi^{2}}{2} Z\left\langle\frac{k T}{\epsilon_{f}}\right\rangle N_{\text {ion }} k T
$$

The last term 18 small for massive WD's and is significant at low mass stars ( $10 \%$ at $\left.0.3-0.4 \mathrm{M}_{\odot} W^{\prime} s\right)$.

Equation of state for WD's Intertor. WD's occupy a certain region in the $(\rho, T)$ plane. The high density limit is determined by general relativistic effects which become dominant at $10 \mathrm{~g} \rho 10$ and by inverse $\beta$-decays which also become important at these densities.

The maximal temperature attained by WD's depends on the pre WD evolution. It has been found that stellar cores with masses smaller than the Chandrasekhar limiting mass cannot ignite $\mathrm{C}^{12}$ and this means that WD's do not stay an appreciable length of time at T (5-6) $\times 10^{8} \mathrm{~K}$.

The $(\rho, T)$ plane can be characterized from the EOS point of view by the following dimensionless parameters:

$$
\begin{aligned}
\mu & =\frac{\epsilon_{E}}{\mathrm{kT}}=\frac{\text { Ferm1 energy }}{\text { Thermal energy }} \\
\beta & =\frac{\mathrm{m}_{\mathrm{e}} \mathrm{c}^{2}}{\mathrm{kT}}=\frac{5.93}{\mathrm{~T}_{9}}=\frac{\text { Electron rest mass energy }}{T_{\text {hermal energy }}} \\
\Gamma & =\frac{(\mathrm{ze})^{2}}{\Gamma \mathrm{kT}}=2.28 \frac{\mathrm{z}^{2}}{\mathrm{~A}^{1 / 3}} \frac{\rho_{6}^{1 / 3}}{\mathrm{~T}_{7}}=\frac{\text { Electrostatic energy }}{\text { Thermal energy }} \\
\Lambda & =\frac{\mathrm{h} \Omega_{\mathrm{p}}}{\mathrm{kT}}=2.240 \frac{\theta}{\mathrm{T}}=0.779 \frac{\mathrm{z}}{\mathrm{A}} \frac{\rho_{6}^{1 / 2}}{\mathrm{~T}_{7}}=\frac{\text { Energy in Lattice vibrations }}{\text { Thermal energy }}
\end{aligned}
$$

The following definitions were used $\bar{r}=\left(3 / 4 \pi n_{\text {ion }}\right)^{1 / 3}$ is the average interparticle distance or the so-called radius of ${ }^{10 n}$ the fon sphere, $\mathrm{n}$ ion the ion number density. $\Omega_{\mathrm{p}}^{2}=4 \pi \mathrm{n}_{\text {ion }} \mathrm{e}^{2} / \mathrm{m}_{\text {ion }}$ is the square of the ion plasma frequency and $\theta$ is the Debye temperature of the solid at these physical conditions.

The discussion of the EOS in the core is limited to elements with atomic weight greater than 4. Pycnonuclear reactions will not allow the existence of lighter elements at these conditions. Also, present day evolutionary tracks towards WD pass through temperatures sufficiently high to burn $\mathrm{H}$ and He before the cooling towards dark WD's begins.

Coulomb fluid. At the densities and temperatures found in the core of WD's the electrons are highly degenerate. At a density of the order of $10^{6} \mathrm{gm} / \mathrm{cm}^{3}$ the Fermi energy of the electrons $1 \mathrm{~s}$ of the order of $\mathrm{m} \mathrm{e}^{2}$. Thus electron interactions can be characterized by an energy of the order of $\frac{1}{2} \mathrm{MeV}$. It is therefore very difficult to perturb the electrons 
and they can be considered as a perfect Fermi gas. When the electrons have such high energies the ions will be fully ionized and we have ions, of charge $\mathrm{Ze}$ moving inside an extremely perfect electron sea. The interaction energy between ions and themselves are characterized by $\Gamma$ which varies from 0.01 to $\sim 200$. The lons hardly perturb the electrons $\left(Z e^{2} / F \mu \ll l\right)$. The electrons serve in this limit only to neutralize the system. The lons themselves are considered as classical particles because the thermal de Broglie wavelength $\lambda_{T}=\left(2 \pi \hbar^{2} / m_{10 n} k T\right)^{\frac{1}{2}}<<F_{\text {. }}$ The matter in the core can therefore be represented as an ane component plasma (OCP). The Coulomb energy of an OCP depends only on the configuration of the ions. Our interest here is in the phase diagram of such a plasma and the EOS throughout the relevant regions in the $(p, T)$ plane. In everyday physical conditions we typify a gas as the physical state in which $\Gamma<<1$; namely, the kinetic energy of the particles is much greater than the interaction energy between them. The solid phase is characterized by $\Gamma \gg 1$. Hence the physical state in the core of WD can be represented as a liquid. The problem of the theoretical model for liquids is that it is usually extrapolated from elther extreme.

Liquids with some short range order are described in terms which correspond to an idealized solid and liquids with ion-1on collisions and without short range order are described by extrapolating the gas model. In the lack of an approprlate model for such liquids numerical methods were resorted to, in particular the method of Monte-Carlo (MC), Molecular Dynamics and in some cases integral equations like the Percus-Yevick equation or the hypernetted chain one.

The OCP poses some serious and special problems because of the infinite range of the interaction and the lack of any typical length. In usual materials the interaction contains a hard-core and has a minimum which defines some length. This length is important to the phase transition between the liquid and the solid. The problem is even more severe, it is questionable whether systems with a long range interaction possess a thermodynamic limit in the sense we know it. Let me mention similar problems in the statistical mechanics of stellar systems and the fact that various thermodynamical ensembles yield different points of instabilities. The thermodynamic IImit of OCP was recently investigated by Lieb and Narnhofer (1975). They proved that a thermodynamic limit does exist. However, the free energy is not a convex function of the density and hence the compressibility can be negative and the grand canonical ensemble is not equivalent to the canonical one.

Our next question is the shape of the phase dlagram and is there a liquid-gas phase transition in an OCP. Recently Cesare et al (1973) have claimed to find a gas-liquid phase transition at $r: \sim 1$. However, as has been shown by Lamb (1974)(L) they used wrong EOS and their "phase transition" is exactly their error. On the other hand, in a gas-Ilquid phase transition the compressibility $(\partial P / \partial V)_{T}$ vanishes. The equation of OCP shows that the compressibility vanishes for $\Gamma{ }_{1}$.

We turn now to the liquid-solid phase transition. Consider first the limit $\Gamma_{+\infty}$. The question is what is the lower bound for the electrostatic energy. Onsager (1939) has shown that the lowest bound can be obtained if the point particles are smeared around the initial points. The best smearing is a uniform charge distribution - namely a sphere of 
radius $\bar{F}$. Hence the lowest bound is given by $-0.9 \mathrm{~N} / \bar{F}$ where $\mathrm{N}$ is the total number of particles. However, the ions are point particles. The crystal with the minimal Madelung energy is found to be the bcc crystal. We give in the table the values of the Madelung energy for the simplest crystals. Note how close the numbers are, in particular the relative difference between $a$ bcc and an fcc crystal is only $6 \times 10^{-5}$. Thus it would seem

\title{
Table 1
}

\author{
crystal \\ fcc \\ bcc \\ Wigner-Seitz sphere
}

Madelung Energies of simple crystals.

$$
\begin{gathered}
E_{M} / 2 \Gamma k T \\
-0.895874 \\
-0.895929 \\
-0.9
\end{gathered}
$$

that at $\mathrm{T}=0$ the OCP will arrange itself in a bcc lattice. However, it is plausible that the various corrections to the OCP will be different by more than the difference in the Madelung energies and the type of crystal formed (and its relevant frequencies) may change. This change, if $1 t$ really happens, is not important to the energy content of the WD.

Having established that the lowest energy state is a bcc crystal we expect a phase change at some finite value of $\Gamma$. Again, since the interaction under consideration does not possess a characteristic length we do not expect a phase change in the usual sense. Namely, the solid phase and the liquid phase resemble each other to a very large degree. The liquid possess a long range order and phonon spectrum which does not differ appreclably from that of the solid. Likewise, the densities of the liquid and the solid are alike since no dramatic change in the packing is expected. Also, the heat of melting will not be high.

At very high densities ground state quantum oscillations will prevent the formation of a crystal. Hence the phase transition line bounds and ends at a finite density.

Coulomb liquid. Recent years have seen much progress beyond the initlal works of Kirzhnits (1960), Abrikosov (1961) and Salpeter (1961). In view of the difficulties encountered in analytic methods resort is made to the MC method developed by Metropolis et al.(1953), Molecular Dynamics and Percus-Yevick and the hypernetted chain (HNC) integral equations. The first MC calculation on OCP was carried out by Carley (1963) and Barker (1965) for $\Gamma \sim 1$ and were extended to a wide range of $\Gamma$ by Brush et al.(1966). Hubbard and Slattery (1971) extended the MC to include the screening effect. This effect is important for hydrogen and helium and will not be discussed here. Later Shaviv and Kovetz (1972)(SK1) and Hansen (1973)(H) have repeated the Brush et al. (1966) (BST) calculation with various assumptions. Recently the method of Integral equations was applied to OCP, in particular by Cooper (1973), Springer et al.(1973) and $\mathrm{Ng}$ (1974) who have solved the HNC equation over a large range of $\Gamma$.

The basic idea of BST is to take a system containing a small number of particles (say 32 to 500) immersed in a uniform neutralizing background. Periodic boundary conditions are used to imitate an 
infinite number of interacting particles. The electrostatic energy between the infinite periodic charges is evaluated by means of the Ewald (1921) sums. The Ewald sum corrects the Ion-ion interaction Inside the cell for the effect of the distant particles. It is easily shown that the effective interaction resembles a spherically symetric screened Coulomb potential plus a small contribution due to nonisotropic slowly varying potential. The expressions for the potentials are still complicated and computer time consuming. Hence BST expanded the potentials into a Taylor series. H remarks that in spite of the fact that the sum in the unisotropic contribution converges very rapidly and only summation of 5 nearest cells is needed to yield an accuracy of $1: 10^{4}$, its calculation is very time consuming. Hence, he expanded the potential in Kubic harmonics and optimized the expansion so as to obtain a relative error in the isotropic part of less than $1 \%$.

SK1 have tried a different tactic. They assumed the $\mathrm{N}$ Ions to be in a sphere. For a given configuration of $\mathrm{N}$ lons in a sphere of radius $R$ filled with a uniform charge $-\mathrm{ZeN}$ the energy is

$$
U=\Gamma k T N^{-1 / 3}\left(-0.9 N^{2}+0.5 N R^{-2} \sum_{i=1}^{N}\left|\vec{r}_{i}\right|^{2}+R \sum_{i \neq j}\left|\vec{r}_{i}-\vec{r}_{j}\right|^{-1}\right)
$$

This expression takes into account the fact that the particles on the surface of this sphere feel only the interaction of the particles inside the sphere. This is obviously_a source of an error in the method. However, the error in $U$ varies like $\mathrm{N}^{-1 / 3}$ and is easily corrected for. The results SK obtained for $\mathrm{N}=100$ and $\mathrm{N}=200$ were used to extrapolate the value of $U$ to $N \rightarrow \infty$. The relative error in $U$ should be less than $1 \%$.

The results are usually expressed in terms of the excess energy defined as:

$$
\Delta U / N k T=\left(U_{\text {coul }}-L i g-U_{\text {bcc }}\right) / N k T
$$

where $U_{\text {coul-liq }}$ and $U_{b c c}$ are the electrostatic energy of the Coulomb liquid coul-liq and bcc the bcc lattice respectively. Figure 1 shows the results obtained by the various investigators. While the total energies are very close to one another ( $0.9 \Gamma$ for $\Gamma \gg 1)$ the energy excess shows deviations. The results obtained according to the HNC integral equation are also shown.

It is of interest to understand the difference between the results and the Implications on cooling WD's. In the MC method of BST and $\mathrm{H}$ a long range order is imposed on the system at all $\Gamma$. All functions are periodic with wavelength greater than L - tne size of the cell. SK do not assume a long range order. An examination of the results of SK indicates that the difference between $N=100$ and 200 is less than $1: 10^{3}$ for $\Gamma>75$ and hence long range order has a small effect.

Another problem with the periodic boundary conditions happens when a particle interacts with a very close image. In the bulk state, such a case does not happen because of mutual repulsion that prevents very short distances. There are MC methods in which such problems are avoided. 
Finally, the number of particles chosen by BST and $H$ is such that it corresponds to an integer number of bcc cells inside the basic calculation unit. SK, who used spherical cells did not constrain the number of particles in the MC in this way.

The HNC method, like to SK1, does not constrain the number of particles and neglects all of the bridge graphs. It is not clear at the moment what is the effect of this neglect on $\mathrm{U} / \mathrm{NkT}$.

In view of the above differences one finds in the literature several fits to the numerical results. H proposed the following expression:

$$
\begin{gathered}
\frac{U}{N k T}=-\Gamma^{3 / 2}\left(\frac{a_{b c c}}{\left(b_{1}+\Gamma\right)^{1 / 2}}-\frac{a_{2}}{\left(b_{2}+\Gamma\right)}-\frac{a_{3}}{\left(b_{3}+\Gamma\right)^{3 / 2}}-\frac{a_{4}}{\left(b_{4}+\Gamma\right)^{2}}\right) \\
\text { where } \quad a_{b c c}=0.895929 \quad b_{1}=4.6664860
\end{gathered}
$$

where

$$
\begin{aligned}
& a_{b c c}=0.895929 \\
& a_{2}=0.11340656 \\
& a_{3}=-0.90872827 \\
& a_{4}=-0.11614773
\end{aligned}
$$$$
b_{2}=13.675411
$$$$
b_{3}=1.8905603
$$$$
\mathrm{b}_{4}=1.0277554
$$

This expression tends to $\mathrm{U}_{\mathrm{bcc}}+\mathrm{a} \Gamma^{1 / 2}$ for $\Gamma \rightarrow \infty$, namely the excess thermal energy goes as $\Gamma^{1 / 2}$. At the $e^{\text {bcc }}$ other extreme, the expression tends to the first term in the DH expansion (Bowers and Salpeter (1960))

$$
U_{D H} / N k T=-\frac{\sqrt{3}}{2} \Gamma^{3 / 2}-\frac{9}{8} \Gamma^{3}\left(\ln \Gamma+0.460+\frac{1}{2} \ln \sqrt{3}\right)
$$

SK1 proposed a simpler expression namely

$$
U / N k T=-a r^{3 / 2} /\left(b+r^{1 / 2}\right)
$$

which tends to the correct limits for $\Gamma+0$ and $\infty$. This formula was used for the interpolation between the calculated points of SKI.

De Witt (1976) found that the following 4 parameter function

$$
U / N k T=a \Gamma+b \Gamma^{S}+c
$$

fits H's data better than eq. (5) with $a=-0.89461 \pm 3 \times 10^{-5}$, $b=0.8165 \pm 8 \times 10^{-4}, c=-0.5012 \pm 1.6 \times 10^{-4}$ and $s=\frac{1}{4}$. The basic idea of the fit is to find the asymptotic behaviour of the thermal part of the internal energy and according to this fit it behaves as $\Gamma^{\mathbb{4} /}$ for $\Gamma \rightarrow \infty$ However, this fit fails for $\Gamma<1$ because it does not reduce to the $D H$ limit. (In H's fit the $\Gamma^{+\infty}$ limit is adjusted to $U_{b c c}$, which is not the case in De Witt's fit).

Finally, the results of the HNC method can be fitted to $U / N k T=-0.900470 \Gamma+0.2688263 \Gamma^{1 / 2}$

$$
+0.07199925 \ln \Gamma+0.0537919
$$

which again does not reduce to the correct limit for $r+0$.

The direct evaluation of $\mathrm{C}_{\mathrm{V}}$ by means of the $\mathrm{MC}$ is possible but not advisable because of the large noise in the results. Consequent- 
ly, consistent thermodynamics is obtained by applying the analytic fits to the electrostatic energy.

The Helmholtz free energy $F$ is given by

so that

$$
F / N k T=\int_{0}^{r}\left(U\left(r^{\prime}\right) / N k T\right) d r^{\prime} / r^{\prime}
$$

and

$$
(P V / N k T)_{\text {coulomb }}=(\Gamma / 3) \partial(F / N k T) / \partial r=U / 3 N k T
$$

$$
\left(c_{v} / N k\right)_{\text {covlomb }}=-r^{2} \partial(U / \Gamma N k T) / \partial r \text {. }
$$

The compressibility is given by

$$
V k_{T} / N k T=-1-\frac{1}{9}\left(-C_{v} / N k+4 U / N k T\right)_{\text {coulomb }}
$$

Here we included the ideal gas contribution to the compressibility $k_{\mathrm{T}}=\mathrm{V}(\partial \mathrm{P} / \partial \mathrm{V})_{\mathrm{T}}$.

The Thomas-Fermi correction (TF). The TF correction is the first correction for the fact that the electrons are attracted by the ions and the real matter deviates from the ideal OCP. The correction is generally small for elements with high $Z$ - which explains why we IImit the discussion to elements heavier than He. SKI derived the following expression for the exchange term:

$$
F_{T F} / N k T=-\frac{e^{2} \Gamma}{2} \partial n_{e}\left(\mu_{0}, T\right) / \partial \mu F^{2} \overline{\operatorname{Var}(\tilde{\phi})}
$$

where $\operatorname{Var}(\phi)=\int\left(\phi-\phi_{a v}\right)^{2} d V \quad \sim$ and the tilda denotes dimensionless quantities defined by $\phi=\tilde{\phi} z \mathrm{e}^{/ F}, V=\tilde{V} N 4 \pi \bar{F} / 3$ and $\widetilde{\operatorname{Var}}(\widetilde{\phi})=\int\left(\widetilde{\phi}-\widetilde{\phi}_{a v}\right)^{2} d \tilde{V}^{.} \quad \phi$ is the Coulomb interaction potential that affects the electrons. The bar implies a canonical average. The various approximations used by different investigators boll down to estimates of $g(\Gamma)=\Gamma \operatorname{Var}(\tilde{\phi})$. SKI used the $\Gamma_{\text {. }}$. S limit for the whole range, namely $g(r)=108 \Gamma / 175$. The $\mathrm{DH}$ theory yields for $\Gamma \rightarrow 0$ $g(\Gamma)=(\Gamma / 3)^{\frac{1}{2}}$. A simple interpolation formula for the whole range of $\Gamma$ is:

$$
g(r)=(108 / 175) r^{1 / 2} /\left(1.070+r^{1 / 2}\right)
$$

$\mathrm{L}$ used a phenomenological expression for $\mathrm{F}_{\mathrm{TF}}$ namely

$$
F_{T F}=-(54 / 175)\left(r^{2} / \beta Z^{2}\right)(\partial \rho / \partial \psi)_{\beta}
$$

which is arranged to fit the $T=0$ EOS of Salpeter (1961).

Quantum ionic correction. The quantum contribution to the ionic free energy can be obtained by perturbation theory (Landau and Lifshitz 1958, §33) namely:

$$
F_{q}=-\frac{\hbar^{2}}{24(k T)^{2}} \overline{\sum\left(\nabla_{i} U\right)^{2} / m_{i o n}}+\frac{\hbar^{2}}{12(k T)^{2}} \overline{\Sigma \nabla_{i}{ }^{2} U / m_{i o n}}
$$

Here $U$ is the electrostatic energy of the 1ons. Generally, the two terms can be combined because

$$
\overline{(\nabla u)^{2}}=\lambda \overline{\nabla^{2} U}
$$

L followed Landau and Lifshitz (1958) and assumed $\lambda=\frac{1}{2}$. However, SK1 
adopted the high $\Gamma$ limit namely the Wigner Seitz expression and hence $\overline{\nabla^{2} U}=0$ (no net charge over an ion sphere). This is the source of the factor of 2 difference mentioned by $L$. The final expression found by SKI is

$$
F_{q} / N k T=1^{2} h(r) / 36 \text {. }
$$

Pollock and Hansen (1973)(PH) used the pair correlation function they obtained from the MC calculations to evaluate $h(\Gamma)$. The WignerSeitz approximation gives $h(r)=3$ while for vmall $r$ the DH gives $h(r) \propto r^{3 / 2}$

This term is a perturbation expansion in powers of $h$ or $\Lambda$. At very high densities $\Lambda>1$ and the expansion diverges. This region is important for the crystallization of heavy elements at high densities. More work has to be done in this case if the evolution of massive WD is to be followed. The contribution of this term can be extremely important. L remarks that if this term is ignored the $\mathrm{T}=0$ solid $\mathrm{C}^{12}$ melts at $\log \rho=6.49$ instead of $\log p=10.7$.

The exchange effect. The contribution of the exchange term of the electrons to the total free energy of the plasma has been first studied In few limits (Zapolsky (1960), De Witt (1969). More recently Kovetz et al. (1972) (KLV) have extended the calculations to the partly degenerate semirelativistic regime and Jancovici (1978) extended the calculations to the near classical limit. KLV provided asymptotic expressions in the various limits and supplied numerical tables for the regions in between.

The exchange term becomes exponentially small in the non-degenerate limit. $\mathrm{KLV}$ found that

$$
F_{\text {ex }} \cong-(\alpha / 4 \pi)\left(m_{e} c^{2} / \pi^{2}\right)\left(V / \lambda_{c}^{3}\right)\left(\pi / \beta^{2}\right) \exp \left(2 \beta\left(\mu-m_{e} c^{2}\right)\right)
$$

for $\beta>1$ (extreme non-relativistic) and $\epsilon_{f}<0$. ( $\mu$ contains the rest mass energy of the electrons). In spite of the fact that the exchange contribution decreases as the gas becomes classical, it has been shown by $L$ that the relative contribution of the exchange is greatest along the boundary between the degenerate and non-degenerate regions. Consequently although the contribution is absolutely only $0\left(\epsilon_{\mathrm{f}} / \mathrm{kT}\right)$ in the non degenerate region it must be included for $\epsilon_{f} \sim 0$.

The crystal state. The ionic crystal state, which corresponds to $\Gamma+\infty$ has two major contributions to the free energy: (a) The Madelung energy, which is the energy of the ground state. (The bcc Madelung energy). (b) The thermal (phonon) part (Kovetz and Shaviv (1970)(KS)).

The contribution of the phonons is calculated in the harmonic approximation, hence the free energy is given by

$$
F / N k T=\sum_{\lambda} \overline{\frac{1}{2} \beta \hbar \omega_{k_{\lambda}}+\ln \left(1-\exp \left(-\beta \hbar \omega_{k_{\lambda}}\right)\right)}
$$

where $\omega_{k \lambda}$ is the circular frequency and is a function of the wave vector $k^{\lambda}$ and the excitation mode $\lambda$. The average is carried out over the first Brillouin zone. With one exception $\omega_{k \lambda}$ is proportional to the ion plasma frequency. The exception is the longitudinal (plasmon) branch at long wavelengths $k^{-1}>k_{s c}{ }^{1}=r_{s c}$, where $r_{s c}$ is the screening radius. In this case we ${ }^{s c}$ have ${ }^{s c}$ (Pines ${ }^{\prime}(1963)$ ). 


$$
\omega^{2}=\Omega_{p}^{2} /\left(1+k_{s c}^{2} / k^{2}\right) .
$$

The magnitude of $\mathrm{k}_{\mathrm{sc}}$ depends on the density. A TF model yields:

$$
k_{s c}^{2}=12 \pi n_{e} e^{2} \lambda(x) / \epsilon_{f} .
$$

KS assumed the relativistic limit to apply and took $\lambda(x)=1$. L applied the semi-relativistic TF model and obtained:

$$
\lambda(x)=\phi(\phi-1) /\left(\phi^{2}-1\right) ; \phi=1+\mu / m_{e} c^{2}
$$

Note that $\lambda(\mathrm{X})+1$ in the relativistic limit and to $\frac{1}{2}$ in the nonrelativistic one. I has also shown that screening is important only at densities below $\log \rho=5.04,5.65$ and 6.25 for $\mathrm{C}^{12}, \mathrm{Mg}^{24}$ and $\mathrm{Fe}^{56}$ respectively.

KS applied the method of Cohen and Keffer (1955) based on $\omega_{k \lambda}$ at 29 points in the first Brilouin zone. The values of $\omega_{k \lambda}$ were also calculated by Kugler (1969) and these were used by $L$.

$\mathrm{PH}$ included for the solid the anharmonic contribution and obtained

$$
\begin{aligned}
& F^{\text {solid } / N k T}=F^{\text {harm } / N k T}-1750 / \Gamma^{2} \\
& \quad=-0.895929 \Gamma+\frac{9}{2} \ln \Gamma-1.8856+\frac{3}{2} \ln \left(\frac{k T}{x_{y}}\right)-1750 / \Gamma^{2}
\end{aligned}
$$

where Ry is the Rydberg energy of the ion. It should be noted that the thermal energy constitutes only $\sim 1 \%$ of the total internal energy of the solid.

Estimated accuracy of the equation of state. The estimated accuracy in the electronic contribution at $\mathrm{T}=0$ are given by Salpeter (1961). Little changes at the relatively low temperatures under discussion.

The error in the ionic contribution is more sensitive to temperature and density. Adding uncertainties of all posstble sources we find a relative error in the free energy of the ions of the order of $1 \%$ or less.

The phase transition. The conditions of phase equilibrium between the solid and the liquid are

and

$$
P_{i s}\left(P_{s}, T\right)+P_{e}\left(P_{s}, T\right)=P_{\text {ill }}\left(P_{l}, T\right)+P_{e}\left(P_{l}, T\right)
$$

$$
\mu_{i s}\left(p_{s}, T\right)+Z \mu_{e}\left(p_{s}, T\right)=\mu_{i l}\left(p_{p}, T\right)+Z \mu_{e}\left(p_{p}, T\right)_{\text {(27) }}
$$

where the subscripts $i, e, 1, s$ refer to lonic, electronic, liquid and solid respectively. The above equations determine $\rho_{\ell}$ and $\rho_{s}$ as a function of $T$ at the transition. $S K$ assumed that $P_{i s}^{\ell}, P_{i 1} \sum_{<} P_{e}$ and $\delta \rho=\rho_{s}{ }^{-\rho_{1}}<<\rho_{s}$ and obtained the following result for ${ }^{1 s^{\prime}}$ the ${ }^{i 1}$ density difference

$$
\begin{aligned}
& \delta \rho=\left(P_{i l}-P_{i s}\right) /\left(\partial P_{i s} / \partial \rho+\partial P_{e} / \partial \rho\right)_{T, P_{l}} \\
& \text { at the phase transition itself is given by }
\end{aligned}
$$

The density at the phase transition itself is given by

$$
f_{\text {is }}\left(P_{l}, T\right)=\operatorname{fil}_{-19-}\left(P_{l}, T\right)
$$


where $f_{i s}$ and $f_{i l}$ are the (ionic) free energy per ion. Note that $\left(\partial \mathrm{P}_{\mathrm{e}} / \partial \rho\right)_{\mathrm{T}}>>\left(\partial \mathrm{P}_{i \mathrm{~s}} / \partial \rho\right)_{\mathrm{T}}$ and that $\left(\partial \mathrm{P}_{\mathrm{e}} / \partial \rho\right)_{\mathrm{T}} \sim \mathrm{P}_{\mathrm{e}} / \rho$. The relative contribution of ionic pressure is $P_{i} / P_{e^{\sim Z}} Z^{2 / 3} \alpha$ and hence

$$
\delta p / \rho \sim z^{3 / 2} \alpha<1 \quad \alpha=137^{-1}
$$

The latent heat release during the phase transition is given by

$$
q=T\left(J_{i s}-J_{i l}\right)=U_{i l}-U_{i s} \text {. }
$$

It is of interest to try and derive the location of the phase transition by other criteria and not only by equating the Gibbs functions (or the free energies). Such a computer experiment was carried out by $\mathrm{PH}$ in the particular case of OCP. The idea is to start a MC calculation in which all particles are at their lattice points and to follow the evolution of

$$
\gamma^{2}=\frac{(\Delta r)^{2}}{d^{2}}=\frac{1}{N} \sum_{i} \frac{\left(\vec{r}_{i}-\vec{R}_{i}\right)^{2}}{d^{2}}
$$

where $d$ is the nearest-neighbour distance in a bcc lattice, $\vec{r}_{i}$ are the instantaneous positions and the $\vec{R}_{i}$ are the equilibrium positions of the $\mathrm{N}$ ions.

PH find that for $\Gamma>160 \gamma^{2}$ remains bounded while for $\Gamma<135 \gamma^{2}$ is not bounded. Hence they argue that $\Gamma=135$ is a lower bound to the melting point. This calculation was however carried out on the OCP in a periodic potential and without additional corrections. A completely different criteria are the various empirical "melting" laws. The best one known is the Lindemann (1910) law which states that the solid melts whenever the parameter $\gamma$ exceeds some critical value. Van Horn (1968). and $\mathrm{PH}$ find for the OCP at the melting point of $\Gamma=160$ that $\gamma=0.17$ which agrees well with the value obtained for other types of fluids with $r^{-n}$ potentlal (Hoover et al.(1970)). Note however that Iindemann's law is based only on the properties of the "solid" and hence cannot be considered as a genuine phase transition.

Some properties of the crystallization curves. If the classical oCP had been the dominant factor in crystallization then $\Gamma$ would have been constant along the phase transition line. However because of the various corrections which do not scale the same way as the classical Coulomb interaction we find deviations from the $\Gamma=$ const line.

Over most of the region the solid and the liquid free energies are very close. Consequently the EOS must be known very accurately to determine $\Gamma_{M}$. An error of $0.1 \%$ in $F$ changes $\Gamma_{M}$ by \pm 10 . L has shown that replacing the bcc Madelung energy by the Wigner-Seitz value (a change of only $10^{-2} \cdot 3$ ) changes $\Gamma_{M}$ to 85 ! (as found by SK). Generally when the crystallization occurs at high densities $\Lambda=2.24 \theta / T>1$ and the crystal is in the Debye cooling region. Actually the "Debye cooling" has already started in the liquid before crystallization.

The latent heat of melting is about $\mathrm{kT}$, very similar to the latent heat of melting of ordinary solids. This small latent heat is a further indication to the very close similarity between the liquid and the solid. 
Let us return for a second to the equation for the compressibility of the 1iquid. We find that for $\Gamma \sim 1$ the compressibility vanishes which indicates that phase transition from gas to liquid could exist just at the place where Coulomb forces "take over". The MC calculations indicate that at this place local order starts to appear in the liquid. However, as discussed previously $U, C_{v}, P$ and $\rho$ are continuous functions throughout this region.

Mixtures. The discussion so far was restricted to pure elements. In reality WD are composed of mixtures. While the most important constituents are $\mathrm{C}^{12}$ and $0^{16}$, small amounts of impurities with various $Z$ are present as well.

The question is how to treat the mixture. Shaviv and Kovetz (1976) (SK2) have analyzed the possible mode of crystallization and have shown that of the three possibilities: a mixture of macroscopic pure element grains, a lattice with sites randomly occupied by $c^{12}$ and $0^{16}$, or an ordered lattice with a distribution determined by the number ratio, the first one is thermodynamically favored. Hence extensive thermodynamic quantities can be written as sums over the two constituents. Equivalently average values of $A^{-1}, Z$ and $Z^{2} / A$ can be used for the crystal phase.

Consider now the liquid phase. Suppose the matter has two species with Ionic charges $Z_{1}$ and $z_{2}$. Define the average charge by $\langle Z\rangle=$ $=x_{1} z_{1}+x_{2} z_{2}$ where $x_{1} \equiv n_{1} / n_{1} n_{1}$ is the number density of specie $i$ and $\mathrm{n}=\sum \mathrm{n}_{\mathbf{i}}$.

The linear $\mathrm{DH}$ theory yields for $\Gamma<<1$ that the internal energy can

$U^{D H}=-\frac{\sqrt{3}}{2}\left(\Gamma\left\langle z^{2}\right\rangle\right)^{3 / 2} ; \Gamma=e^{2} / \bar{r} k T ; \bar{r}=(3 / 4 \pi \eta)^{1 / 3}$

In the high $\Gamma$ limit the Wigner Seltz ion-sphere yields:

$$
u^{W S}=-0.9 r\langle z\rangle^{1 / 3}\left\langle z^{5 / 3}\right\rangle
$$

Extensive HNC calculations by Hansen et a1. (1977) have shown that the excess internal energy of mixing at constant $T$ and charge density,

$$
\begin{gathered}
\Delta U\left(r\langle z\rangle^{1 / 3}, x_{1}\right)=U\left(r\langle z\rangle^{1 / 3}, x_{1}\right)-x_{1} U\left(r\langle z\rangle^{1 / 3}, x_{1}=1\right) \\
-x_{2} U\left(r\langle z\rangle^{1 / 3}, x_{2}=1\right)
\end{gathered}
$$

is negligible compared to $\left.U\left(r_{<z}\right\rangle^{1 / 3}, x_{1}\right)$. Hence the internal energy is given by the sum of the internal energies of the two species calculated with the appropriate parameter:

$U\left(r\langle z\rangle^{1 / 3}, x_{1}\right)=x_{1} U_{0}\left(\Gamma\langle z\rangle^{1 / 3} Z_{1}^{5 / 3}\right)+x_{2} U_{0}\left(r\langle z\rangle^{1 / 3} z_{2}^{5 / 3}\right)$

where $U_{0}(r)$ is the internal energy of the pure OCP. SK2 used a similar expression.

The miscibility of the two fluids in the uniform background depends on the Gibbs free energy of mixing. An evaluation of the Gibbs free energy by Hansen et al.(1977) shows that the two fluids are always 
miscible, in the limit $\bar{r}_{\text {elec }} / a_{0}<<1$ where $\bar{r}_{e l e c}=\left(3 / 4 \pi n_{e}\right)^{1 / 3}$ is the electron sphere. Thus, no separation occurs under WD conditions.

To sumarize, to a very good approximation the thermodynamic properties of the mixture are additive (the solid and the liquid).

The envelope equation of state. The EOS of the envelope is more complicated than the EOS for the interior due to pressure ionization, species of different types (neutrals, molecules, ions, etc.) and complicated interaction between them. The exact EOS is very important for the calculation of the adiabatic gradient and through it the extent of the convective zone and opacity. This in turn affects the central temperature and clearly the $T_{e} \sim T_{c}^{\alpha}$ (or $L \sim T_{c}{ }^{\beta}$ ) relation, where $T_{e}=e$ ffective temperature.

Initial work on the structure of the outer layers of WD's assumed some simplified forms of the Saha equation which were intended for the calculations of the degree of ionization. Several prescriptions were used (Rouse 1964, Stewart and Pyatt 1966 ) to correct the Saha equation 80 as to take into account pressure Ionization, the disappearance of atomic bound states, etc. These models were used for calculations of stellar atmospheres models (cf.Böhm and Grenfell 1973 ). However, it has become clear in recent years (cf. Sweeney 1978 ) that the simple correction of the Saha equation (a) leads to thermodynamic inconsistencies (like negative adiabatic gradients or violation of the Maxwell equations) and (b) The Saha eq. is only an asymptotic expression and the degree of ionization cannot be cast into the form of the Saha equation over the entire $(\rho, T)$ plane.

In view of the above drawbacks the tendency in recent years is to try and derive the EOS in a consistent way (Graboske et al. 1975, Fontaine et al. 1977 , Magni and Mazzitelli 1979). The fdea is to define ${ }^{a}$ model free energy of the system (containing $\mathrm{H}_{2}, \mathrm{H}_{2}^{+}, \mathrm{H}^{-}, \mathrm{H}^{+}, \mathrm{e}^{-}$, $\mathrm{He}, \mathrm{He}^{+}, \mathrm{He}^{++}$and metals) and minimize it. The model free energies assume model forces between any two species and do not derive the forces from first principles.

Once all the interactions (which are themselves of course a consequence of a given state) between all the constituents are given then the so called configurational free energy can be evaluated. Obviously, collective effects are ignored because only pair interactions are assumed.

The internal part of the free energy demands the knowledge of the energy states. These can be calculated assuming some screened potentlal (say DH potentials) as was done by Rogers et al. (1970) (cf. Lovelace and Masson (1962)). Note however that the number of particles in the Debye screening cloud is given by $\sim(3 \Gamma \theta)^{-3 / 2}$ where $\theta=F_{-\frac{1}{2}}(n) / F_{\frac{1}{2}}(n)$ is the correction of the screening radius for electron degeneracy. One has $\theta \rightarrow 1$ for non-degenerate electrons and $\theta \rightarrow 0$ for degenerate electrons. For $r \sim 1, \vartheta \sim 1$ there is not even one particle inside the Debye sphere and the description of the collective interaction in the form of a Yukawa potential is in question. 
Even with this assumption it is impossible to carry out the procedure throughout the whole $(\rho, T)$ plane. Several authors (cf.Grossman and Graboske 1971) have devised free energy models for the whole range. However, it is accurate in a narrow range and becomes increasingly inaccurate outside it. Hence, different approaches (interactions) are assumed for low and high $\rho$ and $T$. At high density most authors use some version of the hot TF model. This collective model is good for $\bar{r} / a_{0}<<1$ or $p>>19 \mathrm{gm} / \mathrm{cc}$. At low density and high $T$ the ideal gas approximation plus small perturbations is used.

These two limits cannot be extended so as to overlap each other because they both fail for $\bar{F} \sim a_{0}$ (interparticle distance $\sim$ Bohr radius). Consequently interpolation between the 1imits is used. A consistent way to construct an interpolated EOS is to interpolate one thermodynamic quantity, the behaviour of which is well established to be monotonic, and derive all others using thermodynamic identities.

Of importance are the estimates of the accuracy in the EOS. The estimates are difficult to make since it is not a genuine perturbation expansion and we cannot estimate the next term. A possible way is to change one of the assumed interactions and find out the effect on $P, \nabla_{a d}$, U, etc. It seems however, that the EOS is accurate to $\sim$ factor of 2. The effect on the structure of the star is however greater. The structure line of low Te WD's envelopes run through most of the problematic region in the $(\rho, T)$ plane and accumulates the error over the whole envelope. Contrary to the situation in other stars where $T_{c}$ is completely insensitive to the structure of the envelope, the central temperature of WD is very sensitive to the structure of the envelope. The error in $\mathrm{T}$ may be greater than a factor of two for WD's with $\mathrm{T}_{\mathrm{e}}<6000^{\circ} \mathrm{K}$. A detailed analysis of the effects of EOS on the structure of ${ }^{2} W D$ is given by Fontaine et al.(1974).

Neutrino energy losses. Theoretical calculations of cooling WD assume generally neutrino loss rates as derived from the hypothesis of a universal Fermi weak interaction, namely the V-A theory for weak interactions. Extensive calculations and very useful expressions for the neutrino losses were given by Beaudet et al.(1967)(BPS). The neutrino processes which are found to be important for WD's are: photo, pair and plasma neutrinos.

In the late sixties Weinberg (1967) and Salam (1968)(WS) have proposed a unified theory of leptons which includes both weak and electromagnetic interactions. The unified theories, of which the wS one is a prototype, include a weak neutral current interaction which can change the matrix elements in the neutrino producing reactions.

A physical point of interest is that the difference between the various theories are difficult to observe in the laboratory and hence it has been suggested that stellar evolution might help in this respect. Dicus (1972) has reevaluated the rate of some neutrino reactions in the new theory. The ratio of the energy losses rate as calculated according to the WS theory to that calculated by BPS according to the universal Fermi interaction depend on the coupling constants $C_{V}$ and $C_{A}$. The results can be summarized as follows: 


$$
\begin{array}{rlrlrl}
\frac{Q \text { (pair, WS })}{Q(\text { pair, BPS })} & =c_{\mathrm{V}}{ }^{2} & \text { for } & \rho / \mu_{e}<10^{5} \mathrm{gm} / \mathrm{cc}, 3 \times 10^{8} \mathrm{~K} \leqslant T \leqslant 3 \times 10^{9} \mathrm{~K} \\
& =\mathrm{c}_{\mathrm{V}}{ }^{2} \text { for } 10^{4} \leqslant p \leqslant 10^{6} \mathrm{gm} / \mathrm{cc}, & T<10^{8} \mathrm{~K} \\
& =\frac{1}{2}\left(\mathrm{C}_{\mathrm{V}}{ }^{2}+\mathrm{c}_{\mathrm{A}}{ }^{2}\right) & \text { for } & \rho \geq 10^{7} \mathrm{gm} / \mathrm{cc}, & T>6 \times 10^{7} \mathrm{~K}
\end{array}
$$

$\frac{Q(\text { photo, WS })}{Q(\text { photo, BPS })}=\frac{1}{2}\left(C_{V}^{2}+C_{A}^{2}\right)$ for extreme relativistic elec. $=\frac{1}{6}\left(C_{v}^{2}+5 C_{A}^{2}\right)$ for non-relativistic non-degenerate

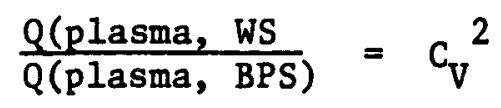

where $C_{A}=\frac{1}{2}$ and $C_{V}=\frac{1}{2}+\frac{e^{2}}{4 m_{w}^{2}} \frac{\sqrt{2}}{G}$. Here $G$ is the usual weak coupling constant, e the electron charge and $m$ the mass of the W-boson. The V-A theory values are recovered for $\mathrm{C}_{\mathrm{V}}=\mathrm{C}_{\mathrm{A}} \mathrm{W}_{1}$. These expressions refer to neutrino losses via electron neutrinos. In the wS theory emission of muon neutrinos cannot be ignored. The expressions for $\nu$ energy losses are 1dentical to $\nu_{e}$ energy losses except for the replacenent of $C_{V}$ by $\mathrm{C}_{\mathrm{y}}{ }^{-1}$ and $\mathrm{C}_{\mathrm{A}}$ by $-\mathrm{C}_{\mathrm{A}}$. The total neutrino losses are given by the sum of the losses due to both types of neutrinos and due to all reactions. The question is the value of $m_{w}$. Present experimental indications are $C_{V} \approx 1.4$ (and $C_{A}=\frac{1}{2}$ ) and the present estimates are:

$$
\frac{Q(\text { pair, WS })}{Q(\text { pair, BPS })} \cong 2 ; \frac{Q \text { (photo, WS })}{Q \text { (photo, BPS })} \cong \frac{1}{2} ; \frac{Q(\text { plasma, WS })}{Q(\text { plasma, BPS })} \cong 2
$$

Thus, the new theory decreases the relative importance of the photo neutrinos relative to the pair and plasma neutrinos. However, the total neutrino loss increases. Recently the role of the neutrino pair bremsstrahlung was evaluated in both theories. Contrary to the previous

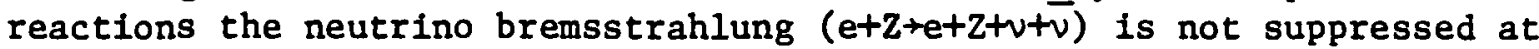
high densities as the various $\nu$ processes are. The bremsstrahlung neutrino reaction is important for WD with medium mass and above.

The process of neutrino bremsstrahlung was first calculated by Gandel'man and Pinaev (1959) for non-relativistic electrons and was extended to relativistic degenerate electrons by Festa and Ruderman (1969). Detailed numerical integrations over a wide range in the $(\rho, T)$ plane were carried out by Cazzola et al. (1971) (see also De Zott1 (1972)). More recently Flowers (1973) has studied extensively all the four neutrino reactions mentioned above and has included several effects, in particular many body effects. In addition Flowers considered the processes In the solid state. Thus the elastic scattering of electrons from the lattice are not suppressed as the density increases and neutrino bremsstrahlung on the static lattice becomes important when all other processes have decayed.

The effects of ion correlations may be quite high-up to a factor of 6. Note that $M C$ calculations show that already for $r \sim 3$ the liquid phase shows a short range order and hence already at such a low $r$ the ion correlation must be taken into account. On the other hand, the extreme case 
of $\Gamma>100$ is absolutely unimportant because all neutrino losses decrease to very small values at this $T$ compared to the photon energy loss.

Dicus et al.(1976) evaluated the effect of the WS theory on the neutrino rates and found

$$
\frac{Q \text { (bremss, WS })}{Q \text { (bremss, BPS) }}=\frac{1}{2}\left(\mathrm{C}_{\mathrm{V}}^{2}+\mathrm{C}_{\mathrm{A}}^{2}\right)
$$

over all the relevant range. The value of this ratio (including muon neutrino losses) is $\sim 1.3$.

Electron conduction. The high heat conductivity by degenerate electrons was established by Marshak (1941) and extended later to the case of semi-degenerate electrons by Mestel (1950) and Lee (1950). These classical theorles assume that the electron is scattered only by the ions and the ions behave independently of one another. This assumption gave rise to divergencles that were eliminated in a rather artificlal way.

Hubbard (1966) introduced the effect of fon correlation and derived the correct cutoff for the Coulomb potential between the ion and the scattered electron. The opacities derived by Hubbard are about a factor of two below the Marshak-Mestel-Lee values. Hubbard's results are essentlally that for $\Gamma<1$ the Debye length should be used as the cutoff parameter, while for $r>20$ the best cutoff length is $1 / 3 F$. The values in the region $1<r<20$ may be obtained by interpolation.

The effect of electron-electron scattering was analyzed extensively by Lampe (1968) and by Hubbard and Lampe (1969). This effect is expected to be small in strongly degenerate matter. However, Lampe (1968) discovered that at the core-envelope interface, where the degeneracy is not strong this effect changes the electron conductivity by up to $50 \%$. Since this is the place where the temperature gradient in the star is determined, this effect is important for the $L-T_{c}$ relation.

Iben (1968) produced extensive tables of electron conductivity opacity assuming that the cutoff is given by the Debye screening radius. Iben (1968) has also examined the effects of uncertainties in the electron conductivity on the structure of the cores of red giants and found them to have very small effects. Finally, he suggested also an analytic approximation to the table which is easy to use on computers.

The extension of the new theory of Hubbard and Hubbard and Lampe to relativistic and semi-degenerate electrons was carried out by Canuto (1970) and Canuto and Solinger (1970) and by Kovetz and Shaviv (1973). However, there seems to be an error in Canuto's reduction of the transport equation. In particular, the results do not reduce to those of Hubbard at low Fermi energies, nor do they have the correct dependence on temperature and density.

Kọvetz and Shaviv (1973), who derived the correct relativisic Boltzmann equation, obtained the electron conductivity in two limits where simple analytic expressions for the pair correlation function is avallable. Thus, they derived the electron conductivity in the 1 imit $\Gamma \ll 1$ 
using the $\mathrm{DH}$ theory and in the limit $\Gamma>75$ using the results from simple cubic lattice.

Now, since the behaviour of the pair correlation function is known, at least numerically, for all $\Gamma^{\prime} s$ no interpolation between the two limits is required.

More recently Flowers and Itoh (1976) have re-evaluated the transport properties of dense matter. They added the effects of impurities and dislocations which influence critically the transport coefficients. The impurities and dislocations limit the transport of heat by providing very efficient scattering mechanisms for electrons and phonons. The contribution of the impurities to the thermal resistance is very sensitIve to the concentration and varies like $(\Delta Z)^{2} X$ where $X$ is the average concentration of the impurities and $\Delta Z$ is the departure of the charge of the impurities from the $Z$ of the most abundant species.

Stevenson and Ashcroft (1974) and Minoo et al.(1976) have revised the Hubbard (1966) and Hubbard and Lampe (1969) results with improved ionic structure factors and electron-ion interaction. While their results are malnly relevant to hydrogen and helium they can easily be extended to higher ionic charges so as to take into account the finite value of $\vec{r}$. They usually find the conductive opacity to be a factor of two lower than the value obtained by Hubbard and Lampe (1969). The electron conduction is important in those regions which are not easily tractable to analytic methods. Thus, the electron conductivity in the solid core is so high that the core is practically 1sothermal. On the other hand, the opacity in general and the conductive opacity in particular are very important at the core-boundary, where $T \gtrsim 5 \times 10^{6}, \epsilon_{f} \sim 0$, p 10. Here pressure ionization is not complete, $r \sim l$ and the assumption of OCP is not a very good one. The very same remark is appropriate to the radiative opacity.

Radiative opacity. The radiative opacity depends on the physical state of the plasma. The knowledge of the "physical state" is equivalent (almost) to the knowledge of the EOS. Hence all problems in the EOS affect the uncertainties in the radiative opacities. A recent review by Carson (1976) covers the general problems of opacity calculations and hence we discuss here briefly only those effects which are characteristic to the dense plasma found in WD's.

Electron scattering is affected by (classical) electron correlations. The reduction in the electron scattering opacity due to correlations was calculated by Diesendorf (1970), Diesendorf and Ninham (1969). The reduction in the opacity is a function of $\Gamma$. For $\Gamma \gg 1$ the reduction reaches a factor of 0.5 (for hydrogen) and 0.7 for He (Aharoni and Opher, 1977, 1979). The reduction for heavier elements is smaller.

The dispersion relation for electromagnetic waves in plasma is $\omega^{2}=\Omega_{p}^{2}+c^{2} k^{2}$ where $\Omega_{p}, \omega$ and $k$ are the ion plasma frequency, the wave frequency and the wave number respectively. Since $\Omega_{p}$ cannot be ignored, the integrand in the Rosseland mean should contain $\left(1-\Omega_{p}^{2} / \omega^{2}\right)$ and the integration should start at $\omega=\Omega_{p} \neq 0$. Cox (1965) ignored this factor in 
the integrand but included it in the range of integration. The effect is a monotonic function of $\Lambda$. For $\Lambda \sim 1$ the corrections are $210 \%$ (increase) to the scattering opacity and $2.5 \%$ (increase) to the absorption opacity (Watson (1969, 1970) Aharon1 and Opher (1979)). While Cox (1965) estimated the effect ot be $O\left(\Lambda^{3}\right)$ for the scattering and $O\left(\Lambda^{6}\right)$ for $\chi$ Kramers opacity, Aharoni (1979) has shown that it is $O\left(\Lambda^{2}\right)$.

The progenitor mass, birth function and luminosity function. The classical assumption made in the construction of the theoretical LF is that all WD's have the same mass and hence are formed from main sequence (MS) stars of the same (average) mass. Furthermore, a constant birth rate is assumed. The WD's observed in the solar neighborhood belong to the disk population. The crucial point is that the cooling time down to a luminosity of $10^{-5} \mathrm{~L}_{0}$ is of the same order as the age of the disk and hence the above assumptions call for correction factors. D'Antona and Mazzitelli (1978) tried to investigate the effect of these assumptions on the LF.

Assume ${ }_{M S}\left(M_{M S}, t\right) d M_{M S}$ is the birth rate of MS stars with masses in the range ( $\left.M_{M S}, M_{M S}+d M_{M S}\right)$. These stars live on the $M S$ and in the post $M S$ phase for $\tau_{M S}\left(M_{M S}\right)$ years and become WD's. In the approximation assumed here $\tau$ depends only on the total mass at the MS phase. The newly formed ${ }^{M D}$ 's cool from high $L$ to low $L$ in time $\tau_{W D}\left(M_{W D}, L\right)$. MS stars with masses ( $\left.M_{M S}, M_{M S}+d M_{M S}\right)$ yield WD's with masses $\left(M_{W^{\prime}} M_{W D}+d M_{W D}\right)$. The function $M_{W D}=M_{W D}\left(M_{M S}\right)$ is known today very poorly. The number of $W D^{\prime} s$ with mass $\left(M_{W D}, M_{W D}+d M_{W D}\right)$ and luminosity $L$ at time $t$ is given by

$$
d N_{\text {WD }}\left(M_{W D}, L\right)=n\left(M_{M S}, t-\tau_{M S}-\tau_{w D}\right) \frac{d \tau_{W D}}{d \log L} d \log L d M_{M S}
$$

The contribution to the LF of these stars is:

$$
L F\left(M_{W D}\right) d M_{W D}=\frac{d N_{W D}}{d \log L}=n\left(M_{M S}, t-\tau_{M S}-\tau_{W D}\right) \frac{d \tau_{W D}}{d \log L} d M_{M S}(38
$$

The observed LF is the integral over all masses, namely:

$$
L F(t, L)=\int_{M_{t 0}}^{M_{\max }} n\left(M_{M S}, t-\tau_{M S}\left(M_{M S}\right)-\tau_{w D}\left(M_{w D}\right)\right) \frac{d \tau_{w D}}{d \log L} d M_{M S}
$$

Here $M_{\text {to }}$ and $M^{0}$ are the mass of the star born with the formation of the disk and max now reaching luminosity $L$ as a cooling WD, and the maximum of the MS star to produce a WD. Since we see only WD's that belong to the disk, suppose the age of the disk is ' $D$ ' than

$$
L F\left(\tau_{D}, L\right)=\text { observed } L F=\int_{M_{t 0}}^{M_{\text {max }}} n\left(M_{M S}, \tau_{D}-\tau_{M S}-\tau_{w D}\right) \frac{d \tau_{w D}}{d \log L} d M_{M S}(40)
$$

The LF has been evaluated in several approximations. The classical approximation assumes $n(M, t)=$ const. and $M_{W D}\left(M_{M S}\right)=\left\langle M M_{D}\right\rangle$, namely all stars produce the same WD. In this approximation we find

$$
L F\left(\tau_{D}, L\right) \propto \frac{d \tau_{W D}}{d \log L}
$$

The results for $\operatorname{LF}(\tau, L)$ obtalned recently by several authors based on (41) are given in figure 2. The application of the full expression for the LF results in changes shown in figure 2 as well. The 
effect of these corrections depends on two questions: (a) to what extent 1s Debye cooling reached before $\mathrm{L}^{2} 10^{-4} \mathrm{~L}_{\Theta}$ and (b) the details of the function $M_{W D}{ }^{-M}\left(M_{M S}\right)$. When Debye cooling is reached before $L \sim 10^{-4} L_{0}$ (a question whlch depends on the EOS In the envelope), the LF starts to bend downward already at $L>10^{-4}-10^{-5} L_{0}$ and the additional reduction in LF at these luminosities is modest. However, if Debye cooling is not reached before $10^{-5} \mathrm{~L}_{\odot}$ the effect can be quite large depending on the age of the disk.

The complications in the LF are not yet over. Studies of WD atmospheres seem to indicate that the masses of non-DA WD's are sys-

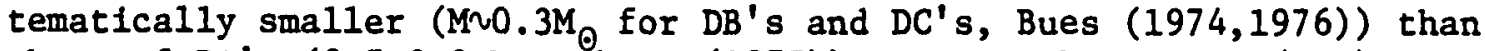

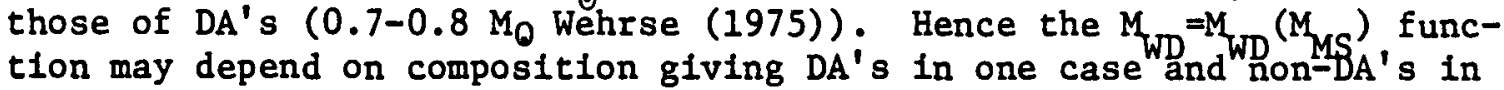
others. Several assumptions are possible. However, in view of the poor knowledge of this function and the intense work at present we leave this question at this point.

Finally all calculations have assumed so far constant $G$. If, as suggested several times, $G$ decreases with time the cooling time increases (Vila 1976) and the paucity of low luminosity WD's will Increase.

The comparison between the theoretical and observational LF's. Kovetz and Shaviv (1976) repeated Weldemann's (1967) derivation of the observed LF of WD's and compared it with the theoretical one. The derivation of the LF was carried out under different assumptions to check the sensitivity of the comparison to the theoretical assumptions.

The conclusions of this work are that the LF is to a very good approximation given by $\log \phi=(0.44 \pm 0.08) \mathrm{M}_{\mathrm{b}}$ down to $\mathrm{m}=15.5$. Various assumptions change the constants slightly but less than the statistical error.

The comparison with theory is not meaningful in the range at which an $L \propto T T^{a}$ with constant a is valid, because the theoretical $L F$ is then given fy: $\log \phi\left(M_{b}\right)=\frac{(a-1)}{2.5 a} M_{b}+$ const. If $a>>1$ the LF is insensitive to $a$, and hence all theoretical models will fit the observed LF equally well.

When Debye cooling sets in the constant a varies quickly and the theoretical LF starts to deviate from a constant slope (in log LF, M plane). Here however, the observational data is very controversial.

We can conclude that the ages of cool WD's are increasingly uncertain as the effective temperature decreases below $110000 \mathrm{~K}$.

Accretion onto WD. The problem of accretion of interstellar gas onto WD has been examined recently by several authors (Castellani and Panagia 1971, Koester 1976, Truran, Starrfield, Strittmatter, Wyatt and Sparks 1977, Mazzitell1 1978). If accretion from interstellar matter is an important effect, then obviously several phenomena can happen: 
(a) The minimal accretion rate will set a minimal luminosity to which WD can cool.

(b) Accretion at sufficiently high rates can remove WD from the cooling sequence, set the WD on a "heating" sequence and confuse the LF.

(c) Accretion 'can destroy the existence of unique compositions.

The rate of accretion depends on the physical conditions in the accreted gas. When the density is low and the cross-section for particleparticle collisions is small, the interstellar gas behaves like indivIdual particles. The accretion rate in the single particle approximation was first derived by Eddington (1926) as

$\dot{m}_{E \alpha d}=\left(1+\frac{E_{\text {grav }}}{E_{h i n}}\right) \pi R_{w D}^{2} v P_{\infty}$

where $E_{\text {grav }}=\frac{G M_{W D}}{R_{W D}}$ and $E_{k i n}=1 / 2 v^{2}$ are the gravitational and kinetic energy per particle, $v$ is the relative velocity between the WD and the gas at infinity and $\rho_{\infty}$ is the density of the gas.

On the other hand, the accretion rate in the fluid approximation is given by the Bondi (1952) interpolation formula:

$$
\dot{m}_{3}=\left(\frac{2 E_{g r a v}}{\gamma E_{t h}+2 E_{h i n}}\right) 2 \pi R_{w D}^{2} P_{\infty}\left(c_{\infty}^{2}+v^{2}\right)^{1 / 2}
$$

where $\gamma$ is the adiabatic exponent of the gas, $E_{\text {th }}$ the thermal energy and $C_{\infty}$ the speed of sound at infinity. The basic idea of Bond1 is that fluid particles are deviated, get to the axis where their transverse momentum is destroyed by collisions and fall back onto the accreting object. The ratio between the two accretion rates does not depend on the density:

$$
\frac{\dot{m}_{g}}{\dot{m}_{E d d}}=\frac{4 E_{\text {hin }}}{\gamma E_{t h}+2 E_{\text {hin }}}\left(1+\frac{c^{2}}{v^{2}}\right)^{1 / 2}
$$

However, the question whether the Eddington particle approximation is good or the Bondi fluid approximation is valid depends on the density (Shara 1977). The critical density is

$$
n_{\text {crit }}=\left(\frac{E_{\text {kin }}}{E_{\text {grav }}}\right) \frac{1}{\sigma R_{W D}}
$$

Consider the typical conditions in the interstellar medium, one finds $\mathrm{n}_{\mathrm{crtt}} \simeq 10^{3} \mathrm{~cm}^{-3}$ (assuming neutral $\mathrm{H}$ ). Consequently, accretion from the generaft interstellar medium should follow the Eddington expression. A similar result was derived by Koester (1976). Mazzitelli (1978) arrived at the same result from the following argument. The accretion rate does not destroy the composition differences between the various WD's. Hence the upper limit to the accretion rate by interstellar gas is

$$
\dot{m}<\left(\frac{m_{a t m}}{\tau_{G}}\right)\left(\frac{Z_{w D}}{Z_{\text {interstellar }}}\right)
$$

where $m_{\text {atm }}$ is the mass in the atmosphere above optical depth of unity,

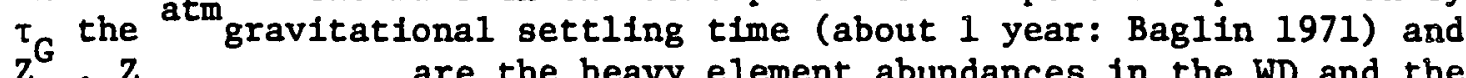
$\mathrm{Z}_{\text {WD' }}^{\mathrm{G}} \mathrm{Z}_{\text {Interstellar }}$ are the heavy element abundances in the WD and the interstellar gas respectively. The substitution of typical values 
$\left(\mathrm{m}_{\mathrm{atm}} 210^{-15} \mathrm{M}_{O}\right)$ yields $\dot{\mathrm{M}}<10^{-3} \dot{\mathrm{m}}_{\mathrm{B}}$ in agreement with eq. (44) for $\dot{\mathrm{m}}_{\mathrm{B}} / \dot{\mathrm{m}}_{\mathrm{Edd}}$. Under these conditions $L_{\text {acc }} \sim 6 \times 10^{-12} L_{\odot}$ and is completely negligible.

The situation changes however if the WD collides with an interstellar cloud. Here particle density can be $n \sim 400 \mathrm{~cm}^{3}$ and the Bondi approximation begins to become valid. The validity of the Bondi approximation is extremely important because the ratio $\dot{\mathrm{m}}_{\mathrm{B}} / \dot{\mathrm{m}}_{\mathrm{Edd}}>1$ and the whole picture changes.

The fate of such a WD depends on the following time-scales:

(a) the thermonuclear runaway time for a given envelope mass $\tau_{\mathrm{N}}$ ' (c) the accretion time-scale $\tau_{\text {acc }} \sim \mathrm{m}_{\text {envel }} / \dot{m}_{\text {acc }} \cdot$ When $\tau_{\mathrm{N}} \ll \tau_{c}$, $\tau_{\text {envel }}$,

thermonuclear runaways will dominate and vice-versa. The problem is, however, that the time-scales are very close to one another. We find $\tau_{\mathrm{N}}{ }^{5 \times 10^{5}}-5 \times 10^{6}$ years, $\tau_{\mathrm{c}} \sim\left(\pi \mathrm{n}_{\mathrm{cloud}} \mathrm{R}_{\mathrm{cloud}}^{2} \mathrm{v}\right)^{-1} \sim 6 \times 10^{8}$ years and $\tau$ envel $\sim 10^{5}-10^{6}$ years. Hence, the exact fate is not clear and a single sengrelova explosion is possible. The next question is how many such events occur to a given WD during 1 ts $10^{9}-10^{10}$ years of cooling to $10^{-4}-10^{-5} \mathrm{~L}_{\Theta}$. The calculation (Truran et al.1977) shows that a typical WD has a probability of $\sim 0.1$ per cooling time to accrete enough material. When this happens the WD moves backwards on the "cooling sequence" unt1l it burns all 1ts hydrogen or becomes a nova. Subsequently the WD returns quickly to its previous location because the core does not absorb much energy. Hence the confusion to the LF is negligible at the present state of the observational material.

Acknowledgement. It is a great pleasure to thank A. Kovetz for collaborating during many years, helpful discussions, useful disagreements and for reading this manuscript.

\section{REFERENCES}

Abrikosov, A. 1961, Soviet Phys. JETP 12, 1254.

Aharoni, U. and Opher, R. 1977, Astron. \& Astrophys. 58, 373

Aharon1, U. and Opher, R. 1979, Astron. \& Astrophys. (In press). Aharoni, U. 1979, M.Sc. Thesis, Israel Inst. of Technology. Baglin, A. 1971, in White Dwarfs ed. Luyten p.136, Reidel Pub. Barker, A. A. 1965, Australian J. Phys. 18, 119. Beaudet G., Petrosian, V. and Salpeter, E. E. 1967, Ap. J. 155, 203. Böhm, K. H. and Grenfell, T. C. 1973, Astron. \& Astrophys. $\underline{28}, 79$. Bond1, H. 1952, M.N.R.A.S. 112, 195.

Bowers, D. L. and Salpeter, E. E. 1960, Phys. Rev. 119, 1180. Brush, S. G., Sahlin, H. L. and Teller, E., 1966, J. Chem. Phys. 45, 2102. Bues, I. 1974, 1st European Workshop on WD, Kiel Untversity Bues, I. 1976, 2nd European Workshop on WD, Rome. Canuto, V., 1970, Ap.J. 159, 641.

Canuto, V. and Solinger, A. B. 1970, Astrophys. Lett. $6,141$. Carley, D. D. 1963, Phys. Rev. 131, 1406. 
Carson, T. R. 1976, Ann. Rev. Astr. \& Astrophys. 14, 95.

Castellan1, V. and Panag1a, N. 1971, Astrophys. \& Space Sc1.,10, 122. Cazzola, P., De Zott1, G. and Saggion, A., 1971, Phys. Rev. D3, 1722. Cesare de L., Forlani, A., and Platania, G。 1973, Ap. and Space Sci. 21, 461 .

Cohen, M. H. and Keffer, F. 1955, Phys. Rev. 99, 1128.

Cooper, M. S. 1973, Phys. Rev. A7, 1.

Cox, A. N. 1965, Stellar Absorption Coefficients and Opacities, chap.3, Stellar Structure, ed. Allen, Chicago Press.

D'Antona, F. and Mazzitell1, I., 1978, Astron. \& Astrophys. 66, 453.

Dicus, D. A. 1972, Phys. Rev. D6, 941.

Dicus, D. A., Kolb, E. W., Schramm, D. N., and Tubbs, D. L., 1976, Ap. J. $210,481$.

Diesendorf, M. O. 1970, Nature 227, 266

Diesendorf, M. O. and Ninham, B. W. 1969, Ap. J. 156, 1069.

De Witt, H. E. 1969, In Low Luminosīty S̄tars, ed. S. Kumar, New York Gordon \& Breach, p.218

De Witt, H. E. 1976, Phys. Rev. Al4, 1290.

De Zott1, G., 1972, Estratto dalle Memorie della Socleta Astronomica Italiana $43,89$.

Ewald, P. P. 1921, Ann. Physik 64, 253.

Eddington, A. S. 1926, in The Internal Constitution of the Stars pp.391-393, Cambridge Univers1ty Press, Cambridge.

Festa, G. and Ruderman, M. A., 1969, Phys. Rev. 180, 1227.

Flowers, E. and Itoh, N. 1976, Ap. J. 206, 218.

Flowers, E., 1973, Ap. J. 180, 911.

Fontaine, G., Van Horn, H. M., Böhm, K. H. and Grenfell, T. C., 1974, Ap. J. 193, 205.

Fontaine, G., Graboske, H. C. and Van Horn, H. M. 1977, Ap. J. Supple. $35,293$.

Gande $\bar{I}^{\prime} \operatorname{man}$, G. M. and Pinaev, V. S. 1959, Zh. Eksper 1 Teoret. F1z. 37, 1072 (Soviet Physics - JETP 10, 764).

Graboske, H. C., Olness, R. J. and Grossman, A. S. 1975, Ap. J. 199, 255.

Grossman, A. S. and Graboske, H. C., 1971, Ap. J. 164, 475.

Hansen, J. P. 1973, Phys. Rev. A8, 3096.

Hansen, J. P., Torrie, G. M. and Vieillefosse, P., 1977, Phys. Rev. A16, 2153.

Hoover, W. G., Ross, M., Johnson, K. W., Henderson, D., Barker, J. A. and Brown, B. C. 1970, J. Chem. Phys. 52, 4931.

Hubbard, W. B. 1966, Ap. J. 146, 853.

Hubbard;'W. B. and Lampe, M., 1969, Ap. J. Supple, 163, 18, 297.

Hubbard, W. B. and Slattery, W. I. 1971, Ap. J. 168, 131.

Iben, I., 1968, Ap. J. 154, 557.

Jancovic1, B., 1978, Phys1ca 91A, 152.

Kirzhnits, D. A. 1960, Soviet Physics - JETP 11, 365.

Koester, D. 1976, Astron。\& Astrophys. 52, 415.

Koester, D. 1978, Astron. \& Astrophys. 64, 289.

Kovetz, A. and Shaviv, G. 1970, Astron. Astrophys. 8, 398.

Kovetz, A., Lamb, D. Q., and Van Horn, H. M. 1972, Ap. J. 174, 109.

Kovetz, A. and Shaviv, G. 1973, Astron. \& Astrophys. 28, 315.

Kovetz, A. and Shaviv, G. 1976, Astron. Astrophys. $52,403$. 
Kugler, A.A. 1969, Annals. Phys. 53, 133.

Landau, L.D. and Lifshitz, E.M. 1958, Statistical Physics, Pergamon Press, London.

Lamb, D.Q. 1974, Ph.D. Thesis, University of Rochester.

Lamb, D.Q. and Van Horn, H.M. 1975, Ap.J. 200, 306.

Lampe, M., 1968, Phys. Rev. 170, 306.

Lee, T.D. 1950, Ap.J. 111, $\overline{625}$.

Lieb, L.H. and Narnhofer, H. 1975, J. Stat. Phys. 12, 291.

Lindemann, F. 1910, Phys. Z., 11, 609.

Lovelace, C. and Masson, D. 1962, Nuovo C1mento 26, 472.

Marshak, R.E. 1941, Ann. N.Y. Acad. Sc1. 41, 49.

Magn1, G. and Mazz1tellf, I. 1979, Astron. Astrophys. 72, 134.

Mazzite111, I. 1978, Laboratorio di Astrophysica Spaziale, (CNR) report No. 26.

Mestel, L. 1950, Proc. Cambridge Ph11. Soc. 46, 331.

Metropolis, N.A., Rosenbluth, A.W. and M.N. and Teller, A.H. and E. 1953, J. Chem. Phys. 21, 1087.

Minoo, H., Deutsch, C. and Hansen, J.P. 1976, Phys. Rev. Al4, 840.

$\mathrm{Ng}, \mathrm{K} . \mathrm{C} .1974$, J. Chem. Phys. 612680.

Onsager, L. 1939, J. Phys. Chem. 43, 189.

Pollock, E.L. and Hansen, J.P. 1973, Phys. Rev. A8, 3110.

Pines, D. 1963, Elementary Excitations in Solids, Benjamin N.Y. p.243.

Rogers, F.J., Graboske, H.C. and Harwood, D.J. 1970, Phys. Rev. A1, 1577.

Rouse, C.A. 1964, Ap.J. 139, 339.

Salam, A. 1967, in Elementary Particle Physles, ed. N. Svartholm (Stockholm, Almquist \& Wiksells).

Salpeter, E.E. 1961, Ap.J. 134, 669.

Shara, M.M. 1977, Ph.D. Thesis, Tel Aviv University.

Shaviv, G. and Kovetz, A. 1972, Astron. \& Astrophys. 16, 72.

Shaviv, G. and Kovetz, A. 1976, Astron. \& Astrophys. 51, 383.

Springer, J.F., Pokrant, M.A. and Stevens, F.A. 1973, J. Chem.

Phys. 58, 5863.

Stevenson, D.J. and Ashcroft, N.W. 1974, Phys. Rev. A9, 782.

Stewart, J.C. and Pyatt, K.D. 1966, Ap.J. 144, 1203.

Sweeney, M.A. 1978, Ap.J. 220, 335.

Truran, J.W., Starrfield, S.G., Strittmatter, P.A., Wyatt, S.P. and Sparks, W.M. 1977, Ap.J. 211, 539.

Van Horn, H.M. 1968, Ap.J. 151, 227.

Van Horn, H.M. 1971, in White Dwarfs, Ed. Luyten, p. 136, Reldel Pub.

Vila, S.C. 1976, Ap.J., 206, 213.

Watson, W.D. 1969, Ap.J. 158, 303.

Watson, W.D. 1970, Ap.J. 159 , 641.

Weldemann, V. 1967, Z. Astrophys. 67, 286.

Weinberg, S. 1967, Phys. Rev. Lett. 19, 1264.

Wehrse, R. 1975, Astron. \& Astrophys. 39, 169.

Zapolsky, H.S. 1960, Cornell University report (unpublished). 


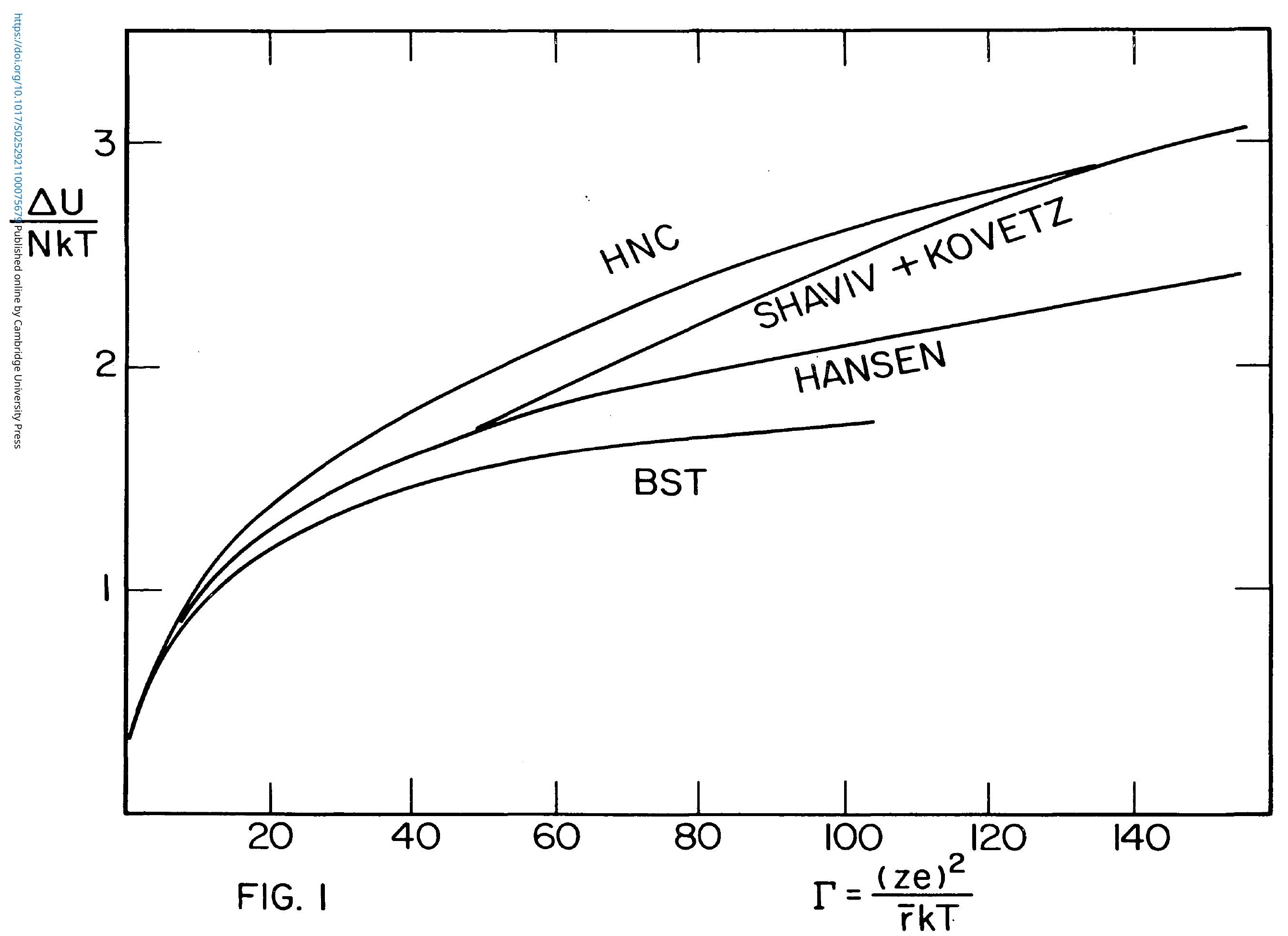




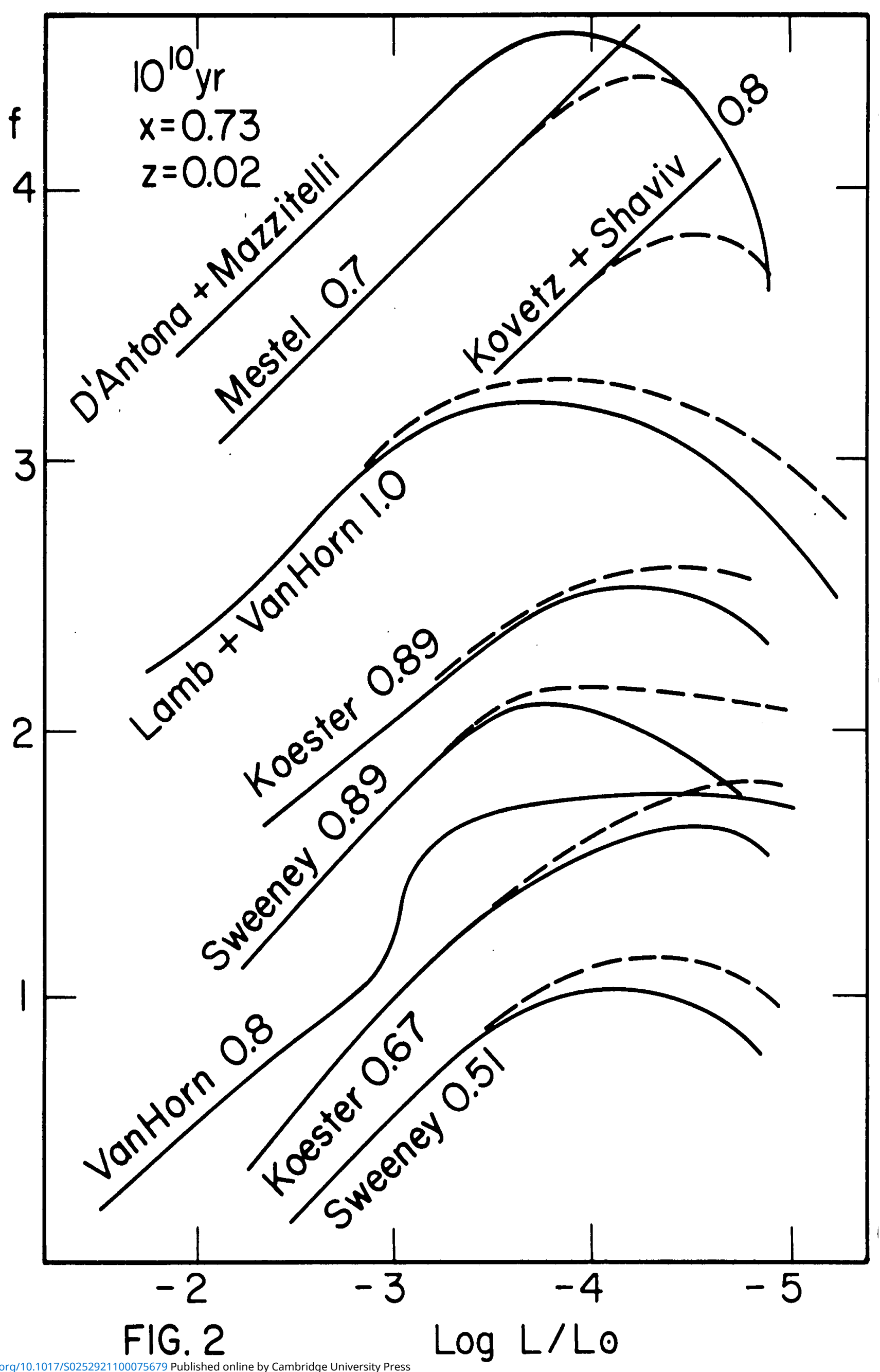

